\title{
Application of Fault Tree Analysis in Supervising Critical Control Points of Spray Dryer
}

\author{
Bao Han \\ School of Software, University of Science and Technology Liaoning, Anshan, China \\ sky46bh@126.com
}

\begin{abstract}
FAT (Fault tree analysis) of drying processing was performed to investigate the causes for faults in equipments management. The causes were deductively identified as the events causing every possible hazard by constructing a fault tree. The fault tree was constructed in a hierarchical structure with a single top event fault, four intermediate event faults, and eight basic event faults connected by a Boolean AND gate, or an OR gate. Qualitative analysis of the fault tree yielded minimal cut sets, structural importance, and common cause vulnerability. Quantitative analysis of the fault tree yielded simulation of final top event fault, cut set importance, item importance, and sensitivity. Basically, these factors were a measure to represent the priority order of the basic events causing the top event. The priority order of these basic events was consistent between qualitative and quantitative analysis. Therefore, the FTA, with qualitative and quantitative analysis, was a good alternative approach to fault analysis in spray dryer, and it also could be further used to the analysis of critical control points..
\end{abstract}

Keywords: Fault Tree Analysis, Drying Processing, Qualitative Analysis, Quantitative Analysis, Critical Control Points, Spray Dryer

\section{Introduction}

FTA (fault tree analysis) [1-3] is a systematic and effective analysis technique for protection against hazards, which can be the additional method in HACCP (hazard analysis and critical control point) [4-6]. Since FTA technique was founded for the first time in the missile launching safety control system in 1962, it has been applied in numerous industrial working fields that require extremely safe and reliable systems [7-12]. Besides, it can also be applied in the fault detection. Compared with HACCP, FTA has the advantage of identifying CCPs (critical control points) quantitatively or qualitatively [13-15], which can increase the efficiency of managing the CCPs. In practice, the CCPs of the basic events may be smaller in scale, or perhaps more detailed and actual than those of the process-scale components. However, the CCPs are mostly given as process-scale components in HACCP, and they should be practically split into more events or more sets of basic events, which are generally controlled according to the safety prescriptions without a priority order. Consequently, the implementation of the supervision of CCPs in HACCP requires further management of the basic events belonging to the CCPs. Therefore, FTA, which can identify the basic events with high risk priorities, would be a supplementary method to fully facilitate the practice of CCPs.

In this paper, FTA was used in the supervision of CCPs of spray dryer [16, 17] with two main purposes: the first was to enhance the effective management of spray dryer in a more systematic and structured order, and the second was to extend the application filed of FTA as a novel technique. All the basic events involved in spray dryer were analyzed quantitatively or qualitatively with various FTA techniques, and the priority order of each basic event was 
estimated. Besides, a CCP management system for spray dryer was built to specify the basic events causing faults in a hierarchical configuration, and the critical basic events were also provided as the control targets.

\section{Preliminaries}

\subsection{Construction of Fault Tree}

A general fault tree analysis can be conducted according to the procedures shown in Figure 1. First, a top event should be defined, and then other events can be identified stepwise by a deductive method. In this method, the cause of a particular event plays as the event of the next hierarchical level, which can be sought based on a literature, survey or expert knowledge. Thus, the cause for the next event can be determined stepwise until the basic events in the lowest hierarchical level of the fault tree are identified.

On the fault tree, all events are correlated with a Boolean logic operator, i.e., an AND gate or an OR gate. The AND gate, equivalent to the Boolean symbol $\otimes$, represents the union of the events combined by the AND gate, which means an event occurs when all the input events in the next hierarchical level combined by the AND gate occur; while the OR gate, equivalent to the Boolean symbol $\oplus$, represents the intersection of the events combined by the OR gate, which means an event occurs when any one or more of the events in the next hierarchical level combined by the OR gate occur.

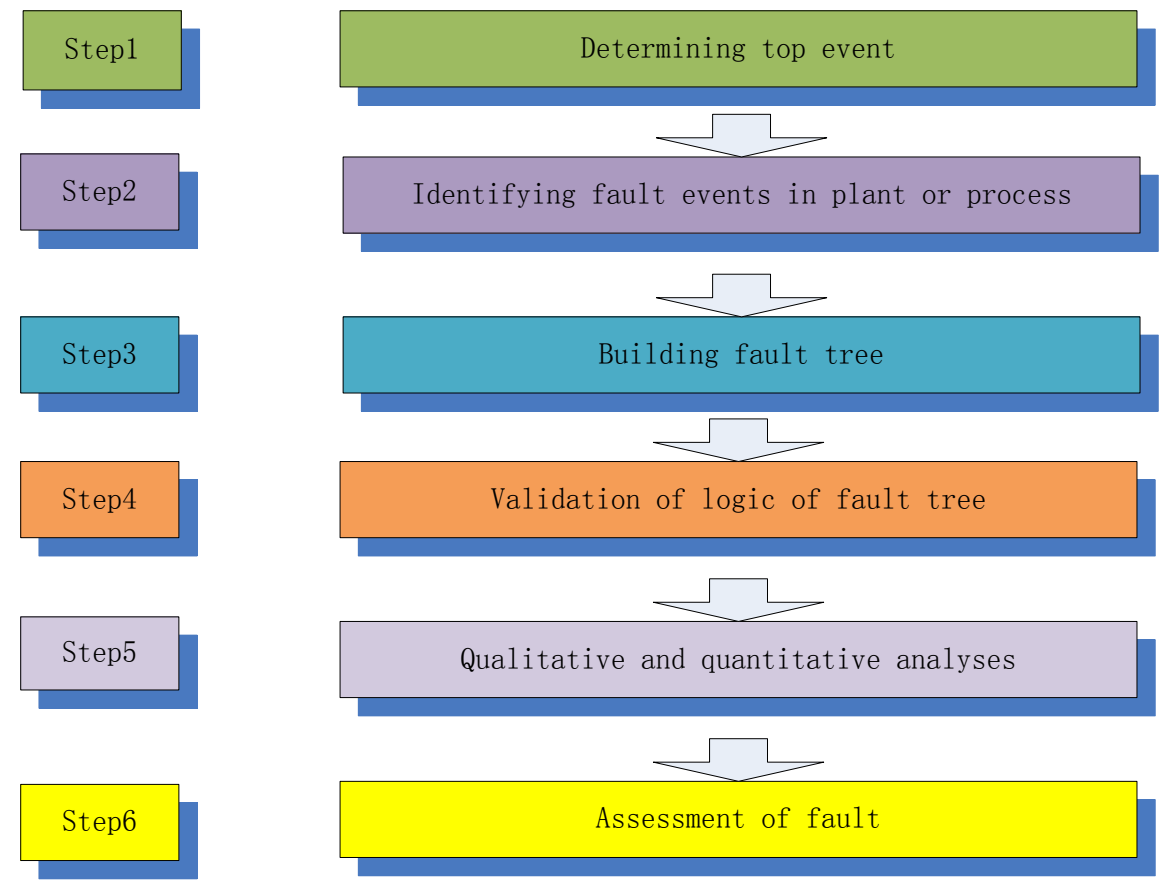

Figure 1. Procedures of Fault Tree Analysis

Figure 2 shows a simple example of fault tree, and a brief description is also given as follows to explain the method. After determining the top event $(C)$, the causes for $(C)$ are sought as the intermediate events $(E)$ and $(F)$. Then, the causes for event $(E)$ are sought as several basic events $\left(X_{1}\right),\left(X_{2}\right)$ and $\left(X_{3}\right)$, and those for event $(F)$ are sought as another intermediate event $(G)$ and the basic event $\left(X_{4}\right)$. Next, the causes for event $(G)$ are the basic events $\left(X_{5}\right)$ and $\left(X_{6}\right)$. Finally, all the fault tree events in different hierarchical structure are 
combined by either an AND gate or an OR gate according to their particular relationships with each other.

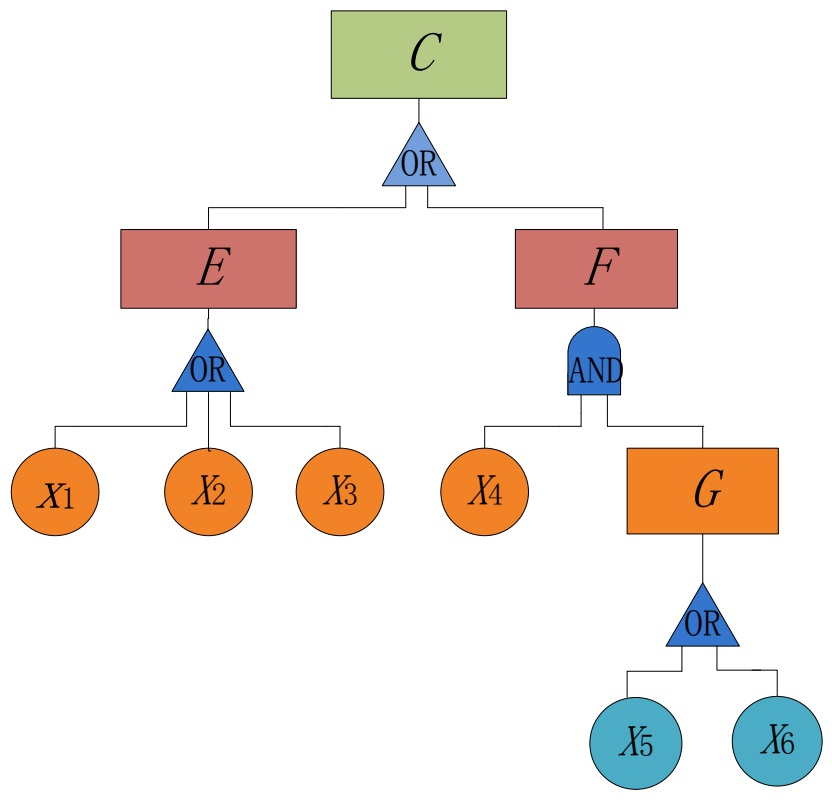

Figure 2. A Simple Example of Fault Tree

\subsection{The Qualitative Analysis}

From qualitative analysis, we can obtain the minimal cut sets, structural importance, and common cause vulnerability.

The cut sets refer to any set of the basic events that cause the top event, and the minimal cut sets refer to the least-needed cut sets that cause the top event. The top event (C) in Figure 2 can be expressed by Eq. (1) with the symbol $\oplus$ for an OR gate as follows:

$$
\text { Top }=E \oplus F
$$

Where $E=X_{1} \oplus X_{2} \oplus X_{3}, F=X_{4} \otimes G$, and $G=X_{5} \oplus X_{6}$. According to the structural correlation in Figure.2, the event (F) is expressed by Eq. (2) as:

$$
F=X_{4} \otimes\left(X_{5} \oplus X_{6}\right)
$$

Thus, the top event (C) can be expressed in terms of the basic events as shown in Eq. (3):

$$
\begin{aligned}
\text { Top } & =E \oplus F \\
& =X_{1} \oplus X_{2} \oplus X_{3} \oplus X_{4} \otimes\left(X_{5} \oplus X_{6}\right) \\
& =X_{1} \oplus X_{2} \oplus X_{3} \oplus\left(X_{4} \otimes X_{5}\right) \oplus\left(X_{4} \otimes X_{5}\right)
\end{aligned}
$$

Eq. (3) can be used to describe the Boolean-indicated cut sets. In this example, the cut sets are equivalent to the minimal cut sets, because there are no more sets left out. In this way, there are five minimal cut sets, which are described as $\{X 1\},\{X 2\},\{X 3\},\{X 4, X 5\}$ and $\{\mathrm{X} 4, \mathrm{X} 6\}$. The minimal cut sets are in OR correlation, and the basic events within the minimal cut sets are in AND correlation.

Structural importance indicates the contribution degree of each minimal cut set to the occurrence of the top event. Assuming the occurrence probability $(P<1)$ of all basic events is equal, multiplication by any probability will reduce the magnitude of the product, whereas addition by any probability will increase the magnitude of the sum. Therefore, a minimal cut 
set with more basic events has much less probability, which indicates a less structural importance degree. Thus, the first group of the minimal cut sets with one basic event $(\{\mathrm{X} 1\}$, $\{X 2\}$ and $\{X 3\})$ is higher in structural importance degree than the second group with two basic events $(\{\mathrm{X} 4, \mathrm{X} 5\}$ and $\{\mathrm{X} 4, \mathrm{X} 6\})$.

Common cause vulnerability indicates the susceptibility of the basic events to an initial common cause. One can define the categories of common causes, and classify the basic events into one of the categories. Then, the number of basic causes gradually lessens to fewer fault origins or common causes. Supposing the basic events shown in Eq. (3) are derived from human errors, then the basic events can be expressed with a single common cause by adding the subscript ' $h$ ' to the corresponding basic events, which is shown as follows:

$$
\text { Top }=X_{1}^{h} \oplus X_{2}^{h} \oplus X_{3}^{h} \oplus\left(X_{4}^{h} \otimes X_{5}^{h}\right) \oplus\left(X_{4}^{h} \otimes X_{6}^{h}\right)
$$

\subsection{The Qualitative Analysis}

In this section, the minimal cut sets acquired from the qualitative analysis are quantitatively evaluated, and the quantitative analysis can provide the simulation of probability, item importance, cut set importance and sensitivity.

The probabilities of the events combined by the Boolean logic operators (OR or AND gates) are used to calculate the probability of the output events in the hierarchical level according to Eqs. (5) and (6).

$$
\begin{aligned}
P_{X_{1}} \oplus P_{X_{2}} \oplus \cdots \oplus P_{X_{n}}= & 1-\prod_{i=1}^{n}\left(1-P_{X_{i}}\right) \\
= & 1-\left(1-P_{X_{1}}\right) \times\left(1-P_{X_{2}}\right) \times \cdots \times\left(1-P_{X_{n}}\right) \\
= & \sum_{i=1}^{n}\left(P_{X_{i}}\right)-\sum_{i}^{n-1} \sum_{j=i+1}^{n}\left(P_{X_{i}} \times P_{X_{j}}\right)+\cdots+(-1)^{n-1}\left(P_{X_{i}} \times P_{X_{j}} \times \cdots \times P_{X_{n}}\right) \\
& P_{X_{1}} \otimes P_{X_{2}} \otimes \cdots \otimes P_{X_{n}}=\prod_{i=1}^{n}\left(P_{X_{i}}\right)=P_{X_{1}} \times P_{X_{2}} \times \cdots \times P_{X_{n}}
\end{aligned}
$$

where $P_{F}$ (fault probability) means the occurrence likelihood of object failures or action errors, and $P_{X_{1}}$ stands for $P_{F}$ of the basic event $\left(X_{1}\right)$ in the arithmetic expression. Thus, the simulation of the probability of the top event in Figure 2 can be calculated by Eq. (7) according to Eq. (3).

$$
P_{\text {Top }}=1-\left(1-P_{X_{1}}\right) \times\left(1-P_{X_{2}}\right) \times\left(1-P_{X_{3}}\right) \times\left(1-P_{X_{4}} \times P_{X_{5}}\right) \times\left(1-P_{X_{4}} \times P_{X_{6}}\right)
$$

Cut set importance $\left(I_{k}\right)$ is defined as the ratio of probability of each minimal cut set to the sum of probability of all minimal cut sets, which is equal to the probability acquired from the simulation of the top event. $I_{k}$ represents the importance of each minimal cut set, and Eqs. (8) and (9) can be used to calculate $\left(I_{k}\right)$ of the minimal cut sets $\left\{X_{1}\right\}$ and $\left\{X_{4}, X_{5}\right\}$ respectively.

$$
\begin{gathered}
I_{k}\left(X_{1}\right)=P_{X_{1}} / P_{\text {Top }} \\
I_{k}\left(X_{4} \otimes X_{5}\right)=P_{X_{4}} \times P_{X_{5}} / P_{\text {Top }}
\end{gathered}
$$

Item importance $\left(I_{\mathrm{e}}\right)$ is defined as the ratio of the probability sum of the minimal cut sets including a particular basic event to that of all minimal cut sets, which also represents the importance of each basic event. 


$$
\begin{gathered}
I_{\mathrm{e}}\left(X_{1}\right)=P_{X_{1}} / P_{\mathrm{Top}} \\
I_{\mathrm{e}}\left(X_{4}\right)=\left[\left(P_{X_{4}} \otimes P_{X_{5}}\right) \oplus\left(P_{X_{4}} \otimes P_{X_{6}}\right)\right] / P_{\mathrm{Top}} \\
=\left[1-\left(1-P_{X_{4}} \times P_{X_{5}}\right)\left(1-P_{X_{4}} \times P_{X_{6}}\right)\right] / P_{\mathrm{Top}}
\end{gathered}
$$

Here, Eq. (10) is used to calculate $\left(I_{k}\right)$ of the basic event (X1), and Eq. (11) is used to calculate $\left(I_{k}\right)$ of the basic event (X4).

Sensitivity ( $\mathrm{S}$ ) is defined as the partial derivative of the probability of a top event at the probability of a particular basic event, which accounts for the importance of the basic events.

$$
\begin{gathered}
S_{X_{1}}=\partial\left(P_{\text {Top }}\right) / \partial\left(P_{X_{1}}\right)=1 \\
S_{X_{4}}=\partial\left(P_{\text {Top }}\right) / \partial\left(P_{X_{4}}\right)=P_{X_{5}}+P_{X_{6}}
\end{gathered}
$$

Eq. (12) is used to calculate $(S)$ of the basic event $\left(X_{1}\right)$, and Eq. (13) is used to calculate $(S)$ of the basic event $\left(X_{4}\right)$. Here, the terms of higher order are excluded, and only the linear terms in Eq. (6) are considered, which is the so-called "rare event approximation". When $P_{X_{i}}<0.1$, the approximation is accurate within about $10 \%$ of the true probability.

\section{Application of FTA in Supervising Spray Dryer}

\subsection{The Fault Tree of Spray Dryer}

Figure 3 shows a simple example of the application of FTA in spray dryer. The top event $X$, i.e., "the dry powder is unqualified", is caused by two intermediate events, which are "faults in spray drying tower' $(A)$ and 'the fault of slush pumps' $(B)$. As the top event $X$ can be caused independently by the occurrence of either the event $(A)$ or the event $(B)$, the events $(A)$ and $(B)$ should be combined by an OR logic gate.

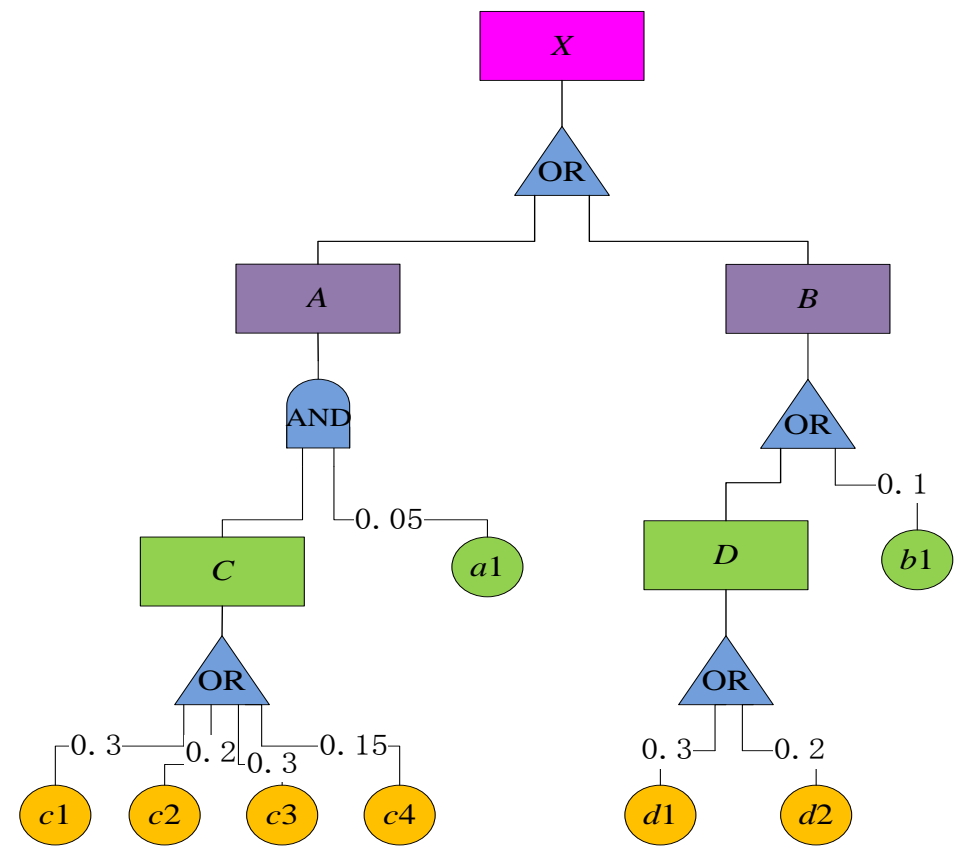

Figure 3. The Fault Tree of Spray Dryer 
For the intermediate event (A) 'faults in spray drying tower', it is caused by an intermediate event and one basic event. The intermediate event is 'the slurry atomization is now ideal' (C), and the basic event is 'the ambient air temperature is too low' (a1). The intermediate event (A) is caused by the combined action of the occurrence of the events (C) and (a1). Thus, the events (C) and (a1) are combined by an AND logic gate. Further, the intermediate event $(\mathrm{C})$ is caused by four basic events, which are 'the fault of filter' (c1), 'the fault of slurry valve' (c2), 'the fault of sealing ring' (c3) and 'the fault of embolism' (c4). These four events can independently act on the occurrence of the intermediate event (C), so they are combined by an OR logic gate.

For the intermediate event (B) 'the fault of slush pumps', it is caused by an intermediate event and one basic event. The intermediate event (D) is 'the fault of sprayer', and the basic event is 'the unreasonable change of the viscosity, density and moisture content of the slurry'. These two events independently act on the occurrence of the intermediate event (B), so they are combined by an OR logic gate. Further, the intermediate event (D) 'the fault of sprayer' is caused by two basic events, which are 'the fault of wafer' (d1) and 'the fault of vortex board' (d2). These two basic events independently act on the occurrence of the intermediate event (D), so they are also combined by an OR logic gate.

Consequently, the whole fault tree of spray dryer can be constructed in a hierarchical structure composed of the top event X, the intermediate events (A) - (D) and the basic events $a_{1}, b_{1}, c_{1}, c_{2}, c_{3}, c_{4}, d_{1}$ and $d_{2}$, as listed in Table 1 .

\section{Table 1. The Basic Events of Faults and their Probability of Accurrence in Spray Dryer}

\begin{tabular}{|c|c|c|}
\hline $\begin{array}{l}\text { The basic } \\
\text { event }\end{array}$ & The description of event & $\begin{array}{c}\text { Fault } \\
\text { probability } P_{F}\end{array}$ \\
\hline$A$ & faults in spray drying tower & \\
\hline$a_{1}$ & the temperature of outgoing air is too low & 0.05 \\
\hline$C$ & the slurry atomization is now ideal & \\
\hline$c_{1}$ & the fault of filter & 0.30 \\
\hline$c_{2}$ & the fault of slurry valve & 0.20 \\
\hline$c_{3}$ & the fault of sealing ring & 0.30 \\
\hline$c_{4}$ & the fault of embolism & 0.15 \\
\hline$B$ & the fault of slush pumps & \\
\hline$b_{1}$ & $\begin{array}{l}\text { the unreasonable change of the viscosity, density and moisture } \\
\text { content of the slurry }\end{array}$ & 0.10 \\
\hline$D$ & the fault of sprayer & \\
\hline$d_{1}$ & the fault of wafer & 0.30 \\
\hline$d_{2}$ & the fault of vortex board & 0.20 \\
\hline
\end{tabular}

\subsection{The Qualitative Analysis}

From qualitative analysis of the fault tree, we can obtain the minimal cut sets, structural importance and common cause vulnerability. 
Taking the fault tree illustrated in Figure 3 as an example, we can describe the logic relationship by Eq. (14) according to Eqs. (1)-(3).

$$
\begin{aligned}
X_{\text {Top }} & =A \oplus B \\
& =\left(a_{1} \otimes C\right) \oplus\left(D \oplus b_{1}\right) \\
& =\left[a_{1} \otimes\left(c_{1} \oplus c_{2} \oplus c_{3} \oplus c_{4}\right)\right] \oplus\left(d_{1} \oplus d_{2} \oplus b_{1}\right) \\
& =\left(a_{1} \otimes c_{1}\right) \oplus\left(a_{1} \otimes c_{2}\right) \oplus\left(a_{1} \otimes c_{3}\right) \oplus\left(a_{1} \otimes c_{4}\right) \oplus d_{1} \oplus d_{2} \oplus b_{1}
\end{aligned}
$$

The fault tree shows four minimal cut sets with four basic events and three minimal cut sets with one basic event, and those minimal cut sets are combined by an OR logic gate. If a minimal cut set is composed of more than one basic event, the basic events are combined by an AND logic gate within the minimal cut set. According to the principle of the minimal cut sets, the top event can be simply perceived as the minimal cut sets in parallel. Furthermore, a particular minimal cut set can be simply identified by considering all the involved basic events.

\subsection{The Quantitative Analysis}

Quantitative analysis can be used to estimate the occurrence probability of top event by simulation, cut set importance, item importance and sensitivity. In order to calculate the above estimations, the fault probability $\left(P_{F}\right)$ of the basic events should be known in advance.

According to the simulation by Eqs. (5)-(7), the fault occurrence probability of the top event is calculated as PTop $=0.4582$, which means there are 4582 faults on 10000 required occasions. If the fault occurrence probability of the basic events PF, is substituted with another value changing with a particular situation in the field. The PTop will change with it.

Cut set importance (Ik), the contribution degree or weight of each minimal cut set to the PTop, can be calculated according to Eqs. (8) and (9). Table 2 shows the calculated results of cut set importance, which agree well with the results from the structural importance analysis. For examples, the most important group is $\left\{b_{1}\right\},\left\{d_{1}\right\},\left\{d_{2}\right\}$, and the lowest important groups is $\left\{a_{1}, c_{1}\right\},\left\{a_{1}, c_{2}\right\},\left\{a_{1}, c_{3}\right\},\left\{a_{1}, c_{4}\right\}$.

Table 2. The Cut Set Importance (Ik)

\begin{tabular}{ccc}
\hline $\begin{array}{c}\text { Minimal Cut } \\
\text { Sets }\end{array}$ & $\begin{array}{c}\text { Cut Set Importance } \\
(\text { Ik) }\end{array}$ & $\begin{array}{c}\text { Structure } \\
\text { importance }\end{array}$ \\
$b_{1}$ & $2.185 E-1$ & High \\
$d_{1}$ & $6.547 E-1$ & High \\
$d_{2}$ & $4.365 E-1$ & High \\
$\left\{a_{1}, c_{1}\right\}$ & $6.547 E-2$ & Low \\
$\left\{a_{1}, c_{2}\right\}$ & $4.365 E-2$ & Low \\
$\left\{a_{1}, c_{3}\right\}$ & $6.547 E-2$ & Low \\
$\left\{a_{1}, c_{4}\right\}$ & $3.273 E-2$ & Low \\
\hline
\end{tabular}


Table 3. The Cut Set Importance $\left(I_{k}\right)$ and Sensitivity

\begin{tabular}{ccc}
\hline $\begin{array}{c}\text { Minimal Cut } \\
\text { Sets }\end{array}$ & $\begin{array}{c}\text { Item Importance } \\
\left(I_{e}\right)\end{array}$ & $\begin{array}{c}\text { Sensitivity } \\
(S)\end{array}$ \\
$b_{1}$ & $2.185 \mathrm{E}-1$ & $1.000 \mathrm{E}+0$ \\
$d_{1}$ & $6.547 \mathrm{E}-1$ & $1.000 \mathrm{E}+0$ \\
$d_{2}$ & $4.365 \mathrm{E}-1$ & $1.000 \mathrm{E}+0$ \\
$a_{1}$ & $2.002 \mathrm{E}-1$ & $9.000 \mathrm{E}-1$ \\
$c_{1}$ & $6.547 \mathrm{E}-2$ & $3.000 \mathrm{E}-1$ \\
$c_{2}$ & $4.365 \mathrm{E}-2$ & $2.000 \mathrm{E}-1$ \\
$c_{3}$ & $6.547 \mathrm{E}-2$ & $3.000 \mathrm{E}-1$ \\
$c_{4}$ & $3.274 \mathrm{E}-2$ & $1.500 \mathrm{E}-1$ \\
\hline
\end{tabular}

Sensitivity $(S)$, the contribution degree of each basic event to the $P_{\text {Top }}$, can be calculated according to Eqs. (12) and (13), as shown in Table 3. It can be seen that the minimal cut sets can be divided into three groups in terms of the sensitivity. The first group is $b_{1}, d_{1}, d_{2}$, the second group is $a_{1}$ and the last group is $c_{1}, c_{2}, c_{3}, c_{4}$.

To sum up, according to the results from structure importance, the cut set importance and the item importance evaluations, the basic events $b_{1}, d_{1}, d_{2}$ and $a_{1}$ should be the critical control points.

\section{Conclusions}

In this paper, FTA (Fault Tree Analysis) was used to create a novel fault management system for the spray dryer. FTA is a method which the causes for fault events are deductively recognized in structured logical manners, and thus setting up a fault tree. The fault tree is built in a hierarchical structure, which includes every possible cause as the structural componets. This fault of spray dryer was divided into much more detailed components that are described in the tree structure. Meantime, the quantitative and qualitative evaluations of the fault tree were analyzed. Eight basic events were considered as the causes for faults in spray dryer. The events on the fault tree were correlated with each other, and several events were recognized as critical control points. According to the analysis of this paper, FTA created a more structured pattern of the management system for the spray dryer. Through FTA, the system faults of spray dryer were divided into as many as eight basic events and their priority for control were also given, which resulting in a novel practical tool for the safety management of spray dryer.

\section{Acknowledgements}

This work was supported by the Nation Nature Science Foundation of China (No.61301095 and No.61201237), Nature Science Foundation of Heilongjiang Province of 
China (No. QC2012C069) and the Fundamental Research Funds for the Central Universities (No. HEUCF1408).

\section{References}

[1]. W. S. Lee, D. L. Grosh, F. A. Tillman and C. H. Lie, "Fault tree analysis, methods, and applications, A review", IEEE Transaction on Reliability, vol. 34, no. 3, (1985).

[2]. W. E. Vesely, F. F. Goldberg, N. H. Roberts and D. F. Haasal, "Fault tree handbook", Washington, DC: Nuclear Regulatory Commission, (1981).

[3]. A. Wild, "The synergistic twins: Fault trees and success trees", Proceedings of the Annual Reliability and Maintainability Symposium, (2005).

[4]. W. Lixing, S. L. J. Ting, W. H. Ip, "Design of Supply-chain Pedigree Interactive Dynamic Explore (SPIDER) for food safety and implementation of Hazard Analysis and Critical Control Points (HACCPs)", Computers and Electronics in Agriculture, vol. 90, (2013).

[5]. B. J. Bobeng and B. D. David, "HACCP models for quality control of entrée production in hospital foodservice systems", Development of hazard analysis critical control point models journal of the American Dietetic Association, vol. 73, no. 5, (1978).

[6]. H. C. Yoo and J. W. Kim, "Development of generic HACCP model for practical application in mass catering establishments", Journal of Korean Society of Food and Cookery Science, vol. 16, no. 3, (2000).

[7]. R. Nait-Said, F. Zidani and N. Ouzraoui, "Fuzzy Risk Graph Model for Determining Safety Integrity Level”, International Journal of Quality, Statistics, and Reliability, (2008).

[8]. N. Jun, T. Wencheng and X. Yan, "A simple algebra for fault tree analysis of static and dynamic systems", IEEE Transactions on Reliability, vol. 62, no. 4, (2013).

[9]. X. Li, J. Zhan, F. Jiang and S. Wang, "Cause analysis of bridge erecting machine tipping accident based on fault tree and corresponding countermeasures", Procedia Engineering, vol. 45, (2012).

[10].M. Xia, X. Li, F. Jiang and S. Wang, "Cause analysis and countermeasures of locomotive runway accident based on fault tree nalysis method", Procedia Engineering, vol. 45, (2012).

[11].Y. Wang, Q. Li, M. Chang, H. Chen and G. Zang, "Research on fault diagnosis expert system based on the neural network and the fault tree technology", Procedia Engineering, vol. 31, (2012).

[12].P. Närman, U. Franke, J. König, M. Buschle and M. Ekstedt, "Enterprise architecture availability analysis using fault trees and stakeholder interviews", Enterprise Information Systems, vol. 8, no. 1, (2014).

[13].M. Bertolini, A. Rizzi and M. Bevilacqua, "An alternative approach to HACCP system implementation", Journal of Food Engineering, vol. 79, no. 4, (2007).

[14].L. Sala, J. Sanz and D. Forestier, "An approach to the analysis of the critical control points of the costa brava water reclamation facilities", Water Practice and Technology, vol. 8, no. 3, (2013).

[15].O. Olufemi and O. Francis, "Improving sachet water quality - does Hazard Analysis and Critical Control Points apply?", Water and Environment Journal, vol. 5, no. 2, (2013).

[16].H. Ting-shun, L. Bao-yu, Z. Bo-qun and S. Li-feng, "Analysis on alarming and common problems of spray dryer", Polyvinyl Chloride, vol. 37, no. 9, (2009).

[17].L. Chaixin, "A case of High Pressure Piston Pump Breakdown's Analysis and Revamp”, Zhejiang Chemical Industry, vol. 44, no. 1, (2013). 
International Journal of Control and Automation Vol. 7, No. 12 (2014) 